\title{
List of the Reviewers of Journal of Dhaka Medical College - 2013
}

1. Prof. Md. Margub Hussain

2. Prof. HAM Nazmul Ahasan

3. Prof. Md. Enamul Karim

4. Prof. Kazi Tarikul Islam

5. Prof. Md. Azizul Kahhar

6. Prof. Md. Ismail Khan

7. Prof. Shamim Ara

8. Prof. Selina Ahmed

9. Prof. Ferdousi Islam

10. Prof. M Ekhlasur Raman

11. Prof. Md. Abid Hossain Mollah

12. Prof. Md. L. E. Fatmi

13. Prof. K. Z. Mamun

14. Prof. H I Lutfar Rahman Khan

15. Prof. Faruk Ahmed

16. Prof. M A Mannan

17. Prof. S H Khundkar

18. Prof. Md. Shahiduzzaman

19. Prof. Sheikh Sader Hossain

20. Prof. Mohiuddin Ahmed Khan
21. Prof. Md. Mahbubul Alam

22. Prof. SM Mahbub Alam

23. Prof. A F Mohiuddin Khan

24. Prof. Jamal Nizamuddin Ahmed

25. Prof. Mansoor Habib

26. Prof. Ehsan Mahmud

27. Prof. Md. Mizanul Haque

28. Prof. Husne Ara Begum

29. Prof. M A Hashem Bhuiyan

30. Dr. Faizul Islam Chowdhury

31. Dr. Md. Golam Kibria

32. Dr. Md. Billal Alam

33. Dr. Iffat Ara

34. Dr. Salma Afroz

35. Dr. Sayeeda Anwar

36. Dr. Md. Azharul Haque

37. Dr. Md. Shamsuzzaman

38. Dr. Abdullah Al-Mamun

39. Dr. Md. Abdul Based

40. Dr. Md. Raziul Haque

41. Dr. Mirza Azizul Haque 